\title{
Shoulder Joint Infectious Arthritis and Acromioclavicular Joint Osteomyelitis due to Candida
}

\author{
Kill-Byung Lim, M.D., Yee-Gyung Kwak, M.D. ', Young-Sup Kim, M.D., Kyung-Rok Park, M.D. \\ Departments of Rehabilitation Medicine and ${ }^{1}$ Internal Medicine, \\ Inje University, College of Medicine, Ilsan Paik Hospital, Goyang 411-706, Korea
}

\begin{abstract}
Candida species inhabit the skin and mucous membranes of healthy individuals with low virulence, and osteomyelitis due to candida is very rare. However, the incidence of invasive candidal infection caused by intravenous drug use, broad-spectrum antibiotics, and indwelling central venous catheter is increasing. A 73-year old man visited the outpatient clinic complaining of right shoulder pain that radiated to the right acromioclavicular joint. He had undergone multiple injection procedures followed by nonsteroidal antiinflammatory drug therapy for several weeks. The ultrasonographic findings showed a heterogeneous mass around the right acromioclavicular joint, while the right shoulder MRI and the overall findings of the body bone scan were suggestive of osteomyelitis. Pathologic findings of ultrasonographically guided joint aspiration fluid showed acute and chronic nonspecific inflammation, while the tissue culture and staining revealed Candida parapsilosis.
\end{abstract}

Key Words Candida, Osteomyelitis, Shoulder pain

\section{INTRODUCTION}

Osteomyelitis is an intractable disease characterized by progressive inflammatory destruction and new bone growth. ${ }^{1}$ It is caused mostly by Staphylococcus aureus and Mycobacterium tuberculosis, and quite rarely by

Received August 19, 2011; Accepted October 8, 2011

Corresponding author: Young-Sup Kim

Department of Rehabilitation Medicine, Inje University, College of Medicine, Ilsan Paik Hospital, 170, Juhwa-ro, Ilsanseo-gu, Goyang 411706, Korea

Tel: +82-31-910-7440, Fax: +82-31-910-7746, E-mail: remedios1004@ naver.com

(c) This is an open-access article distributed under the terms of the Creative Commons Attribution Non-Commercial License (http:// creativecommons.org/licenses/by-nc/3.0) which permits unrestricted noncommercial use, distribution, and reproduction in any medium, provided the original work is properly cited.

Copyright (c) 2012 by Korean Academy of Rehabilitation Medicine candida. ${ }^{2}$ Osteomyelitis caused by candida may be diagnosed late because in many cases, it does not manifest such typical symptoms as pyrexia. Moreover, in many cases it presents non-specific laboratory findings. ${ }^{3}$

We encountered a case of shoulder joint infectious arthritis and acromioclavicular joint and humeral head osteomyelitis occurring secondary to infection with candida after injection treatment. This report now reviews the case with review of the relevant literature.

\section{CASE REPORT}

A 73-year old male patient with pain in the right shoulder joint and acromioclavicular joint was admitted via this hospital's outpatient rehabilitation clinic. He had 
received spinal fusion of L5 and S1 vertebrae 10 years ago for herniation of an intervertebral disc, and was on medication for hypertension diagnosed 10 years ago. He had no history of other medical conditions such as diabetes, tuberculosis, and hepatitis. Further, he had no family history of these particular findings and he had no history of a recent injury. He was a farmer.

A year before the visit to this hospital, he had received several sessions of acupuncture treatment for pain in his right shoulder joint at an oriental medicine hospital. As the pain persisted despite the treatment, he received several sessions of physical therapy, and drug therapy including injections for several months at a nearby orthopedic hospital. For two weeks before the visit to this hospital, pyrexia, skin flare, and edema developed in his right shoulder joint and acromioclavicular joint area with pain radiating from the right shoulder joint to the acromioclavicular joint. Upon presentation to this hospital, his vital signs were stable. However, during a physical examination, a tender point was detected by palpation in the right shoulder joint and acromioclavicular joint.

The patient had a pain score of 9 out of 10 on the visual analogue scale, and the pain worsened through the night. The movement range of the right shoulder joint was limited to $120^{\circ}$ (flexion), $90^{\circ}$ (abduction), $40^{\circ}$ (external rotation), and $45^{\circ}$ (internal rotation), but the range was normal for extension and adduction. Obtaining an accurate assessment of the manual muscle test was impossible due to the pain; however, the results of the sensory function and deep tendon tests were normal. The results of Lhermitte and Spurling tests were negative, and the empty-can test, lift-off test, and Hawkins-Kennedy tests could not be performed due to the pain.

At a routine blood test performed upon presentation to this hospital, the white blood cell level was $6.62 \times 10^{3} /$ $\mu \mathrm{l}$ indicating normal differentiation. Red blood cell sedimentation rate and C-reactive protein level were both high $(52 \mathrm{~mm} / \mathrm{h}$, and $1.2 \mathrm{mg} / \mathrm{dl}$, respectively). No abnormal findings were observed in the other biochemical tests. The results of the ANA, anti-dsDNA, VDRL, RA factor, HLA-B27, and HIV tests, together with the sputum culture test, acid-fast bacillus stain, acid-fast bacillus culture test, nontuberculous mycobacterial test, and mycobacterium tuberculosis polymerase chain reaction test were all negative.

A simple radiography of the right shoulder joint showed a narrowed acromion, sclerotic changes, and bony erosion of the acromioclavicular joint (Fig. 1-A, B). A bone scan using 99mTc DPD showed an increased uptake of radioactive isotope in the humeral head and acromial area of the right shoulder joint (Fig. 2).

Ultrasonography showed the shadow of a large amount of heterogeneous cystic tissue extending from the right subacromial bursa to the right humeral head area (Fig. 3-A, B). On ultrasonography, the right supraspinatus muscle and biceps brachii muscle were not observed. To decompress the enlarged cystoma, $33 \mathrm{cc}$ of cystic fluid was suctioned using a $50 \mathrm{cc}$ syringe under the guidance of ultrasonography.

The cystoma fluid was a red mucus containing yellow
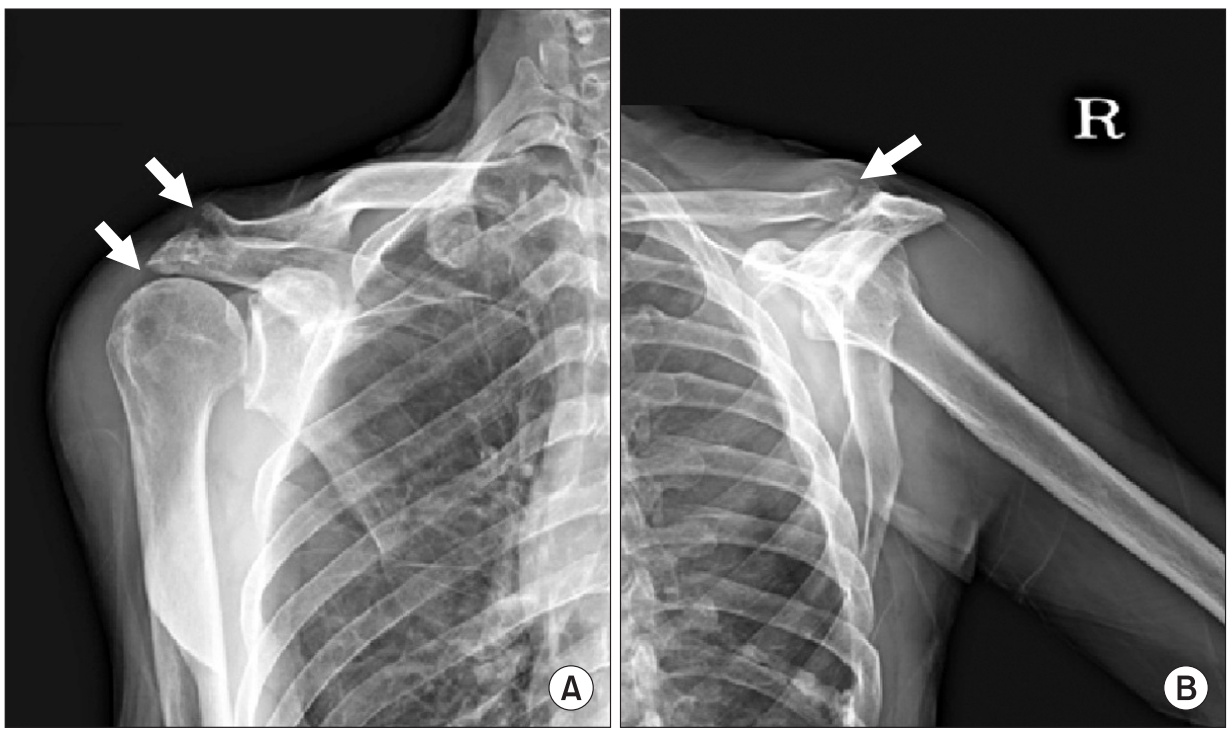

Fig. 1. (A) Anteroposterior radiograph of the right shoulder shows markedly erosive and sclerotic changes in the acromioclavicular joint, and joint space narrowing in the acromiohumeral joint. (B) Y-view radiograph of the right shoulder shows markedly erosive and sclerotic changes in the acromioclavicular joint. 


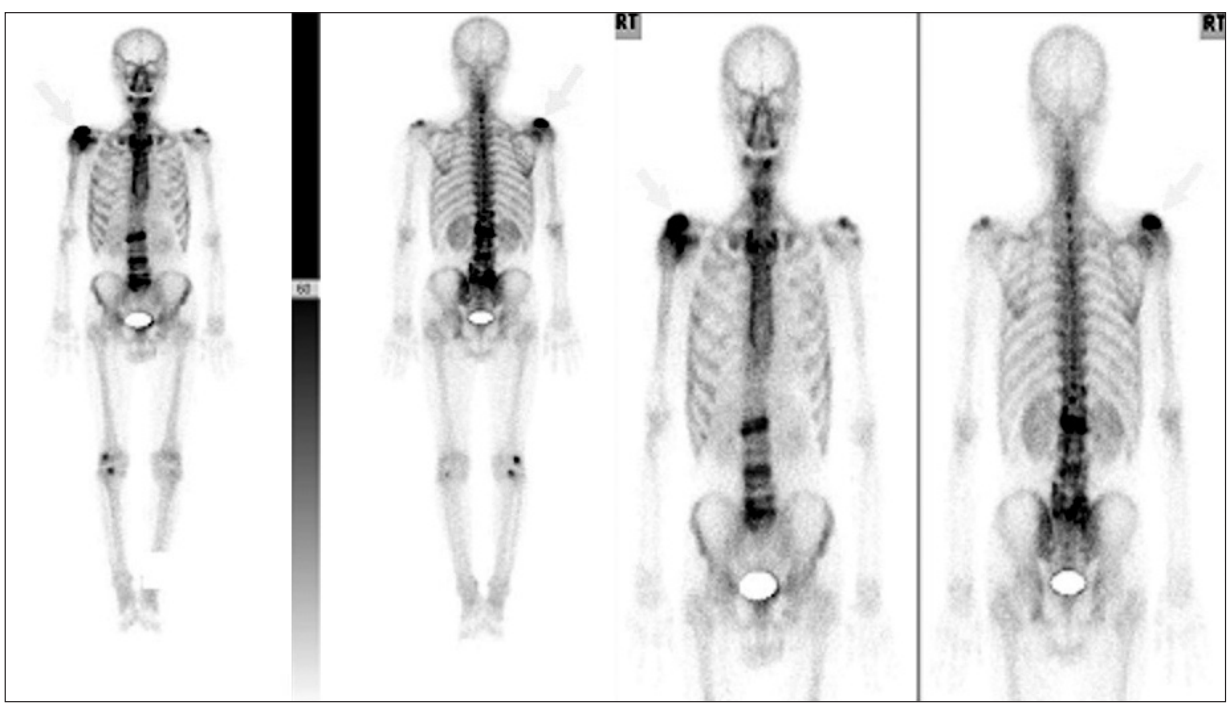

Fig. 2. Whole body bone scan with Tc-99m DPD shows increased radioisotope uptake in the acromial area of the right shoulder.
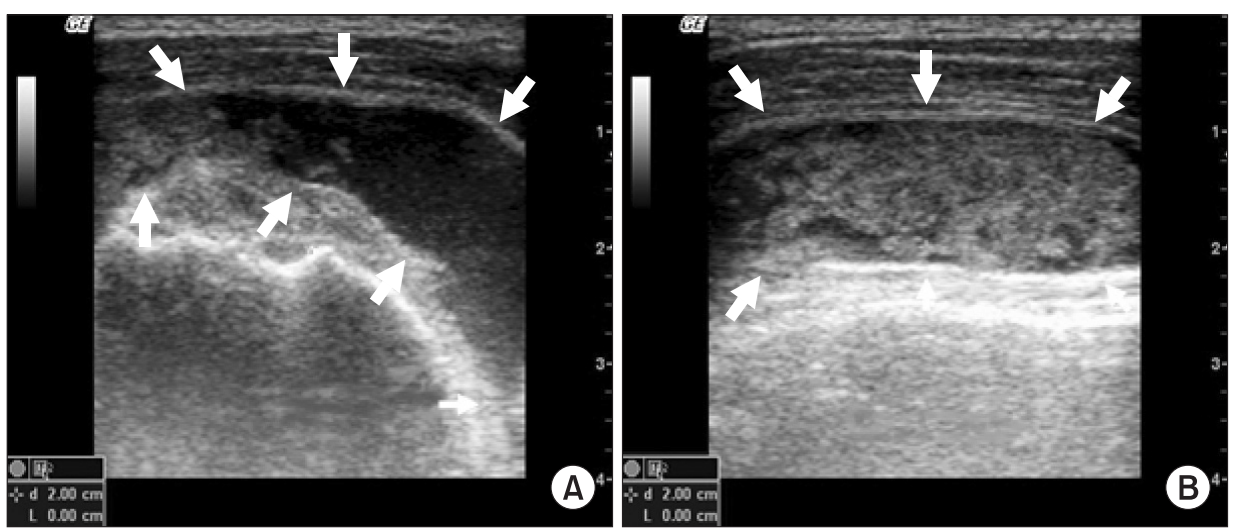

Fig. 3. (A) Short-axis ultrasonographic image of the right humeral head shows a heterogeneous cystic mass around the subacromial bursa. (B) Long-axis ultrasonographic image of the right humeral head shows a heterogeneous cystic mass over the biceps tendon.
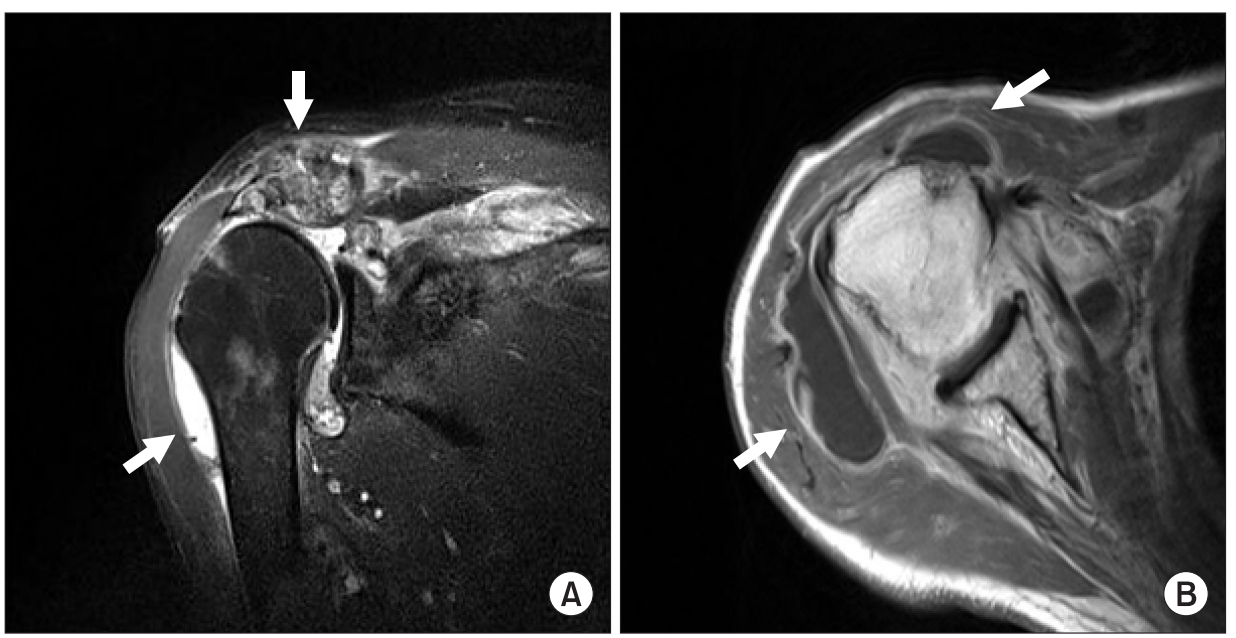

Fig. 4. (A) Transverse T2 weighted magnetic resonance imaging demonstrates joint erosion with osteomyelitis of the acromioclavicular joint and right shoulder joint. (B) Coronal T1 weighted magnetic resonance imaging shows a rim-enhancing lesion in the right shoulder joint compatible with right shoulder joint infectious arthritis.

precipitate. Cytology showed that the red blood cell level was $43,200 / \mathrm{ul}$, white blood cells was $22,000 / \mathrm{dl}$, multinuclear white blood cells were $55 \%$, and lymphocytes were
45\%. Candida parapsilosis was detected in the culture test.

Magnetic resonance imaging of the right shoulder joint 
showed a large amount of effusion, diffuse synovial hypertrophy, contrast enhancement in the shoulder joint, a change in signal intensity, and contrast enhancement in the bone marrow of the acromioclavicular joint (Fig. 4-A). The supraspinatus muscle was not observed in the subacromial space; muscles and ligaments of the long head of the biceps brachii muscle were not observed in the humeral head area. Local change with high signal intensity in the bone marrow and contrast enhancement were observed in the humeral head (Fig. 4-B).

At day 17 of admission, the patient was referred to the orthopedic department where he received arthroscopic irrigation and debridement of the right shoulder joint. During surgery, rupture and pus were found in the biceps brachii muscle and supraspinatus muscle. PCR for Mycobacterium tuberculosis was negative but Candida parapsilosis was detected in the pus culture test.

After intravenous administration of fluconazole 400 $\mathrm{mg}$, the symptoms improved. Three weeks after administration, the VAS score was 1 . A biochemical blood test performed one month after the admission showed that the red blood cell sedimentation rate and the C-reactive protein level had decreased to $32 \mathrm{~mm} / \mathrm{h}$ and $0.2 \mathrm{mg} / \mathrm{dl}$, respectively, without particular symptoms or pain. The patient is now on follow-up.

\section{DISCUSSION}

Candida is normal flora that exists in small numbers in the respiratory system, oral cavity, vagina, and intestinal tract. It can cause opportunistic infections in people with decreased immunity but not in normal people. ${ }^{4}$

Edmond et al. ${ }^{5}$ report that candida has emerged as the $4^{\text {th }}$ most common pathogenic fungus in opportunistic infections in the U.S. because of the recent increase in organ and stem cell transplantation, immunosuppressive treatment, acquired immune deficiency syndrome, cancer, old age, and the use of broad-spectrum antibiotics. Infections caused by candida can occur anywhere in the body. It was reported that in $15 \%$ of the patients with organ infection due to candida, the bone marrow was invaded. ${ }^{6}$ The most common pathogenic fungus is known to be Candida albicans, followed by Candida tropicalis, Candida parapsilosis, and Candida glabrata. ${ }^{6,7}$ However, Candida parapsilosis has rarely been detected as a pathogenic fungus of osteomyelitis and infectious arthritis as it was in this case report.

Studies have reported that most of the patients with osteomyelitis caused by candida had a history of using broad-spectrum antibiotics and receiving central vein cannulation. ${ }^{8}$ An estimated $80 \%$ of the patients had a history of abdominal surgery or parenteral-drug abuse. ${ }^{6}$ The patient in this case report had no history similar to that in previous cases except a history of regular acupuncture treatment for pain in the right shoulder joint that occurred a year ago. Osteomyelitis caused by candida occurs infrequently and presents with atypical symptoms, thus making diagnosis difficult. ${ }^{8}$

Destruction and erosion of bone can be detected by simple radiography, though they can be observed only when lesions are at an advanced stage. In addition, bacterial osteomyelitis may characteristically show swelling in deep soft tissue, bone resorption at the metaphysis, and periosteal reaction. However, osteomyelitis caused by candida rarely presents with these characteristics. ${ }^{9}$ As osteomyelitis caused by candida does not manifest specific clinical symptoms suggestive of the pathogenic bacteria or specific radiographic features, biopsy is required for definite diagnosis. ${ }^{6}$ Medical treatment of osteomyelitis by the administration of the appropriate antifungal agent is essential regardless of surgical treatment. ${ }^{3}$ Currently, amphotericin B and fluconazole are commonly used as drug of first choice; however, there are many dissenting views regarding the therapeutic dose of these drugs and treatment duration., ${ }^{3,6}$

In addition, difficulties in selecting therapeutic agents have been reported because of an increasing number of cases in which candida species-other than Candida albicans-developed resistance to these agents. ${ }^{10}$ In the present case, simple rotator cuff injury was diagnosed as the cause of the pain and treated thus. The pain, however, worsened gradually, and therefore we suspected joint infection. Culture test and further assessment led to a definite diagnosis of shoulder joint infectious arthritis and acromioclavicular joint and humeral head osteomyelitis.

Candida infection may cause osteomyelitis, though less commonly than many bacteria. Therefore, if symptoms of osteomyelitis are manifested, it is important to identify the etiology using a careful diagnostic approach.

Cases such as the present one in which candida caused both osteomyelitis and infectious arthritis have been reported very rarely and never in South Korea prior to 
this case. Opportunistic infections caused by candida are expected to increase in South Korea as the number of patients with decreased immunity is increasing due to an increase in the use of broad-spectrum antibiotics, the ageing of the population, and an increase in organ transplantation in South Korea. Because of this, more studies investigating candida infection in the local population are required. Above all, an understanding of the risk factors and increased index of suspicion of the disease are necessary for the diagnosis of osteomyelitis caused by candida. We experienced a case in which shoulder joint infectious arthritis as well as acromioclavicular joint and humeral head osteomyelitis were caused by candida, a rare cause of shoulder joint pain.

\section{REFERENCES}

1. Waldvogel FA, Papageorgiou PS. Osteomyelitis: the past decade. N Engl J Med 1980; 303: 360-370

2. Lew DP, Waldvogel FA. Osteomyelitis. Lancet 2004; 364: 369-379

3. Arias F, Meta-Essayag S, Landaeta ME, Capriles CH, Perez C, Nunez MJ, Carvajal A, Silva M. Candida albicans osteomyelitis: case report and literature review. Int J Infect Dis 2004; 8: 307-314

4. Kim HJ, Lee JC, Shin BA, Song HY. The Korean society for microbiology textbook of medical microbiology, 4th ed, Killiney: Elsevier Pte Ltd, 2005, 669-676

5. Edmond MB, Wallace SE, McClish DK, Pfaller MA, Jones RN, Wenzel RP. Nosocomial bloodstream infections in United States hospitals: a three-year analysis. Clin Infect Dis 1999; 29: 239-244

6. Cone LA, Byrd RG, Potts BE, Wuesthoff M. Diagnosis and treatment of Candida vertebral osteomyelitis: clinical experience with a short course therapy of amphotericin B lipid complex. Surg Neurol 2004; 62: 234237

7. Petrikkos G, Skiada A, Sabatakou H, Antoniadou A, Dosios T, Giamarellou H. Case report. Successful treatment of two cases of post-surgical sternal osteomyelitis, due to Candida krusei and Candida albicans, respectively, with high doses of triazoles (fluconazole, itraconazole). Mycoses 2001; 44: 422-425

8. Seok H, Kim SH, Kim DH, Kim TH, Kim HK. Cervical osteomyelitis and radiculopathy due to Candida: a case report. J Korean Acad Rehab Med 2007; 31: 482485

9. Pennisi AK, Davis DO, Wiesel S, Moskovitz P. CT appearance of Candida diskitis. J Comput Assist Tomogr 1985; 9: 1050-1054

10. Kim DH, Lee JA, Jo HS, Park KR, Park JD, Kim BI, Choi JH. Systemic candidiasis in neonatal intensive care unit: a 8-year experience. J Korean Soc Neonatol 2001; 8: $33-45$ 